\title{
Estratégias de Navegação em Fórmulas Matemáticas na Web para Pessoas com Deficiência Visual
}

\author{
Hérlon Manollo Cândido Guedes \\ Universidade Federal de Lavras \\ Lavras, MG \\ manollo.guedes11@gmail.com
}

\author{
André Pimenta Freire \\ Universidade Federal de Lavras \\ Lavras, MG \\ apfreire@dcc.ufla.br
}

\begin{abstract}
O desenvolvimento e aprimoramento de recursos de Tecnologia Assistiva como leitores de tela tem possibilitado a pessoas com deficiência visual uma maior independência no que diz respeito à construção de conhecimento e até mesmo em tarefas triviais do dia a dia. Entretanto, o uso dessas tecnologias para aprendizado e atuação profissional em algumas áreas de conhecimento ainda carecem de um maior cuidado. Em particular, ainda há dificuldades para uso de conteúdos de Matemática devido à complexidade das notações com diferentes significados e contextos, o que acaba sendo um desafio aos usuário com deficiência visual. Além disso, ainda há recursos interativos limitados para dar suporte a estratégias de leitura e navegação para este tipo de conteúdo na Web. Desta forma, o presente estudo propõe a elaboração de estratégias para navegação não visual em conteúdos matemático na Web por software leitor de telas para pessoas com deficiência visual, considerando maior liberdade para exploração de diferentes partes do conteúdo de forma não linear. $\mathrm{O}$ estudo propõe a análise e levantamento de pontos positivos e negativos de pesquisas encontradas na literatura, além de buscar propôr uma nova abordagem nos dados levantados na revisão de literatura e em entrevistas com usuários. O trabalho também apresenta uma proposta de avaliação da estratégia através de análise da usabilidade e experiência do usuário, considerando o desempenho nas tarefas e análise de aspectos hedônicos por medição psicofisiológica, como frustração, estresse e engajamento.
\end{abstract}

\section{ACM Classification Keywords}

H.5.2. Information Interfaces and Presentation (e.g. HCI): User Interfaces

\section{Author Keywords}

Deficiência Visual; Matemática; Tecnologia Assistiva; Navegação não visual

Permission to make digital or hard copies of all or part of this work for personal or classroom use is granted without fee provided that copies are not made or distributed for profit or commercial advantage and that copies bear this notice and the full citation on the first page. Copyrights for components of this work owned by others than the author(s) must be honored. Abstracting with credit is permitted. To copy otherwise or republish, to post on servers or to redistribute to lists, requires prior specific permission and/or a fee. Copyright 2018 SBC.

IHC 2018, Anais Estendidos do XVII Simpósio Brasileiro sobre Fatores Humanos em Sistemas Computacionais

Outubro 22-26, 2018, Belém, Brasil

WTD-IHC

\section{INTRODUÇÃO}

O acesso à informação por pessoas com deficiência visual é algo fundamental para o exercício de suas atividades cotidianas. O principal recurso de Tecnologia Assistiva utilizado por essas pessoas é o leitor de tela - software que faz a interpretação do conteúdo na tela e disponibiliza para o usuário por meio de sintetizador de voz.

Atualmente existe um vasto leque de opções de leitores de telas com suporte para vários tipos de sistemas. Dentre estes é possível citar, por exemplo, os leitores NVDA (NonVisual Desktop Access) ${ }^{1}$, JAWS (Job Access With Speech) ${ }^{2}$ e ChromeVox ${ }^{3}$. Estes leitores são amplamente utilizados no mercado para as mais variadas funções. Porém, mesmo que seu uso seja extremamente difundido e sejam capazes de auxiliar em inúmeras tarefas, ainda assim existem limitações consideráveis a serem estudadas e melhoradas. Uma dessas limitações é relacionada à leitura e navegação conteúdo matemático, particularmente com suporte para a língua Portuguesa.

A Matemática possui em seu escopo diversas notações, formas de leitura e significados representativos distintos dependendo da área de estudo da mesma. Por essa razão, devido sua natureza abstrata e sua linguagem bastante simbólica, a compreensão matemática não se faz simplesmente através de uma análise nos elementos separadamente, mas apoiando-se numa análise geral do conteúdo que está sendo lido [13]. Todo este processo neuro-visual, ainda que difícil é feito de forma natural ao efetuar a leitura de uma fórmula matemática por pessoas sem deficiência visual. Isto ocorre devido à utilização da macro visão do conteúdo da fórmula. A macro visão, permite ao leitor inferir um significado a cada elemento da fórmula mediante a fórmula como um todo. Porém, para uma pessoa com deficiência visual este processo pode apresentar dificuldades. Nos moldes atuais das Tecnologias Assistivas, os usuários não possuem condições favoráveis para a realização da visão geral da fórmula. Sendo obrigados a recorrer à leituras lineares e repetitivas e à memorização do conteúdo buscando uma melhor compreensão. Esta repetição e a grande carga de informações que precisam ser memorizadas podem impactar negativamente na compreensão do conteúdo da fórmula.

As páginas Web são desenvolvidas através de marcações propostas por elementos em HTML (HyperText Markup Lan-

\footnotetext{
${ }^{1}$ Disponível em https://www.nvaccess.org/

${ }^{2}$ Disponível em https://www.freedomscientific.com/Products/Blindness/JAWS

${ }^{3}$ Disponível em http://www.chromevox.com/
} 
guage), que seguem estruturas do XML (eXtended Markup Language). Outros tipos de conteúdo, como o matemático são marcados por linguagens baseadas em XML como o MathML. Linguagens orientadas a marcação, como as descritas, favorecem a implementação de recursos de navegação entre elementos. Porém, ainda que a navegabilidade possa ser explorada, é importante realizar investigações sobre estratégias de navegação. Atualmente, existem relatos na literatura a respeito de estratégias de navegação que pessoas com deficiência visual usam na Web $[15,5]$. No caso de navegação em fórmulas matemáticas, a literatura é bastante limitada. Além disso, no caso específico do Brasil, existe um limitador sério que é a ausência de suporte à leitura satisfatória de fórmulas matemáticas em língua Portuguesa nos principais leitores disponíveis no mercado.

Dessa forma, a proposta deste trabalho é um estudo visando o desenvolvimento de estratégias para auxiliar usuários com deficiência visual na navegação em conteúdo matemático, de forma a obter uma compreensão melhor da estrutura de fórmulas e das relações entre seus componentes. O desenvolvimento das técnicas será realizado por meio de estudos na literatura, estudos com usuários por meio de entrevistas, implementação de protótipo seguida de avaliação com usuários.

\section{TRABALHOS RELACIONADOS}

Com a crescente tecnológica e avanços científicos envolvendo pesquisas relacionadas ao desenvolvimento de recursos de Tecnologia Assistiva (TA), diversas propostas tem surgido de modo a melhorar a qualidade de vida e trazer a independência à pessoas com deficiência. Especificamente, voltando-se para o auxílio à pessoas com deficiência visual, algumas tecnologias como leitores de tela e linhas braille foram estudadas, criadas e aprimoradas ao longo do tempo. Todavia, algumas limitações ainda necessitam de maior atenção e estudo, como o tratamento da interação com fórmulas matemáticas, por exemplo.

Devido ao amplo uso de elementos visuais nas notações, complexidade dos conteúdos e variação de significados dos elementos de acordo com o contexto, a transmissão do conteúdo matemático para meios não visuais torna-se um grande desafio [14].

Alguns estudos voltados ao uso de leitores de tela na interação matemática foram desenvolvidos ao longo do tempo. Muitos deles abordaram uma proposta voltada à navegação do conteúdo matemático em forma de árvore de expressão $[6,3,12,11,2]$. Esta abordagem é atualmente implementada pelos leitores de tela JAWS e ChromeVox. Porém, alguns trabalhos evidenciam a fragilidade da utilização deste modelo de navegação $[14,12]$, sendo um dos obstáculos enfrentados a necessidade de conhecimento prévio sobre estruturas de árvore para uma boa interação com este tipo de navegação.

Outros sistemas ainda voltam seus esforços a uma navegação linear [1,4]. Dentre estes pode-se citar o NVDA. A navegação linear feita pelo NVDA se dá por meio da integração com um plugin externo, de modo que além de ser linear não oferece qualquer tipo de interação com o usuário. O plugin em questão é o MathPlayer $[9,10,11]$. Apesar de o autor afirmar possuir várias formas de navegação [9], na integração com o NVDA, só é possível navegar para frente e voltar para o texto anterior.

Para pessoas sem deficiência visual, a leitura de conteúdo matemático permite contar com uma visualização global (overview) e estratégias para direcionar a atenção para elementos específicos. Porém, para pessoas com deficiência visual que usam software de navegação linear um esforço maior é necessário, já que a audição não permite a obtenção da visão geral, e o conteúdo é transmitido de maneira linear. Deste modo, por muitas vezes é necessário ao usuário ouvir repetidamente o conteúdo até ter uma compreensão considerável daquilo que foi transmitido. $\mathrm{O}$ desgaste mental e frustração causados pela necessidade de repetição, aliado com o grande volume de dados que precisa ser armazenado mentalmente pelo usuário colaboram para que a compreensão matemática seja prejudicada. Ao analisar os resultados obtidos por Edwards et al. [1], é possível notar uma possível tendência dos usuários por preferirem abordagens munidas de alternativas diferentes em contraposição às possibilidades puramente lineares.

De fato, estudos anteriores mostraram que a utilização de recursos de navegação baseados em elementos estruturais colabora com a melhoria de desempenho na Web. No estudo de Watanabe [15], por exemplo, foi verificado que o uso de navegação por cabeçalhos em páginas longas auxiliava de forma positiva a obtenção de noção do conteúdo das páginas para melhorar a navegação. $\mathrm{O}$ estudo de Power et al. [5], também reforça o benefício trazido por este tipo de estratégia na navegação.

Existe ainda na literatura alguns estudos envolvendo análises comparativas dos métodos de navegação propostos pelos principais leitores de tela do mercado $[8,7]$. Através destas análises é possível concluir que ainda existem aspectos possíveis de serem explorados para fornecer uma melhora no que diz respeito ao esforço de navegação e consequentemente na experiência dos usuários através de uma evolução nos métodos de navegação matemática.

Em um dos estudos anteriores [7], feito por membros do grupo de pesquisa do autor deste trabalho, foram feitas análises com modelos de tarefas com propostas preliminares para navegação em elementos com raíz, fração e somatório. Apesar de ainda não ter consolidado estratégias de navegação, os resultados projetados foram positivos e contribuíram para sugerir uma primeira evidência inicial da viabilidade da proposta deste projeto.

\section{METODOLOGIA}

\section{Entrevista com pessoas com deficiência visual}

$\mathrm{O}$ trabalho envolveu entrevistas realizadas com usuários em potencial, visando levantar dificuldades enfrentadas no aprendizado da matemática por pessoas com deficiência visual nas escolas regulares e estratégias utilizadas para a transmissão de conteúdo matemático nas aulas. Foram abordadas também questões envolvendo o uso de leitores de tela para leitura matemática, pontos críticos e também sugestões de melhorias.

A entrevista contou com doze participantes de idades entre 9 e 65 anos. Dentre eles participantes com cegueira congênita, cegueira não congênita, baixa visão congênita e catarata 
congênita. Quanto a escolaridade participaram pessoas com ensino superior completo, ensino superior incompleto, ensino médio e fundamental.

O modelo utilizado foi entrevista semi-estruturada, pela flexibilidade na obtenção de dados e possibilidade de enfoque nas questões primordiais ao trabalho, como as já citadas.

As entrevistas foram precedidas da apresentação e assinatura de um termo de consentimento. No termo constava o projeto ao qual se destinava as questões, o motivo da participação do entrevistado, entre outras informações relevantes. O termo também tratava sobre o sigilo da entrevista. Além disso, o entrevistado era instruído sobre sua liberdade de interrupção da entrevista além de possibilidade de esclarecimento de qualquer dúvida a qualquer momento.

\section{Análise de técnicas de navegação na literatura}

Conforme descrito na seção Trabalhos Relacionados, existem alguns estudos envolvendo técnicas de navegação em conteúdo matemático [1, 4, 6, 3, 12, 11, 2]. Porém, ainda é necessário realizar mais pesquisas sobre o tema e verificar a adequação das técnicas para navegação.

Sendo assim, prentede-se realizar uma revisão sistemática da literatura a fim de evidenciar estratégias de navegação envolvendo conteúdo matemático e estratégias de navegação em páginas Web em geral. Pretende-se também levantar pontos fortes e fracos das estratégias atuais sobre conteúdo matemático especificamente. Assim, através deste estudo objetiva-se analisar a possibilidade da aplicação de conceitos envolvendo a navegação voltada à outros aspectos para dentro do universo matemático dos leitores de tela. Além disso, com os possíveis pontos fortes e fracos das estratégias de navegação matemática atuais, será possível selecionar aspectos que podem ser continuados e lacunas que precisam ser sanadas.

Desse modo, a partir de todo este levantamento espera-se realizar a implementação de um protótipo de navegação utilizando o ChromeVox.

\section{Implementação de protótipo com recursos de navegação utilizando o ChromeVox}

A partir de todo o estudo feito, espera-se aliar as informações obtidas na revisão de literatura às evidenciadas nas entrevistas. Com isso, elaborar uma nova abordagem que seja capaz de minimizar ou eliminar as lacunas evidenciadas pelo estudo. Deste modo, espera-se ser possível a implementação de um protótipo de navegação sobre o ChromeVox, a fim de validar a proposta de navegação levantada pelo trabalho.

A escolha pelo ChromeVox se deu pelo fato de ser um leitor de telas de código aberto, além de possibilitar a manipulação da forma com que é trabalhada a estrutura de uma página web. O leitor de telas NVDA, por exemplo, apesar de ser mais amplamente utilizado, faz leitura de fórmulas com plugin de terceiros que não tem código disponibilizado abertamente.

A implementação de leitura em Português está em realização, no contexto do projeto NavMatBR, no qual este projeto se insere. O projeto utiliza técnicas de Processamento de Língua
Natural, com análise de leituras de fórmulas feitas por professores de matemática brasileiros que atuaram no ensino de estudantes com deficiência visual.

A linguagem de marcação que será considerada para a implementação do software é o MathML. A escolha pelo MathML deve-se ao fato de ser uma linguagem de marcação de padrão XML recomendada pelo $\mathrm{W} 3 \mathrm{C}$, cujo objetivo é exatamente a descrição de notações matemáticas. Além disso, os leitores de tela que tem suporte para leitura de fórmulas atualmente são implementados com base em MathML.

\section{Testes de usabilidade com tarefas de navegação em fór- mulas com diferentes níveis de complexidade}

Uma vez que a implementação do protótipo tenha sido feita, será possível aplicá-lo a um cenário de testes controlado. Os testes deverão ser realizados utilizando metodologias bem fundamentadas na literatura para a avaliação de usabilidade de sistemas computacionais.

Para a realização dos testes deverão ser selecionadas pessoas com diferentes níveis de experiência com leitores de tela, deficiência visual e conhecimento matemático. Sendo assim, inserindo o sistema a um ambiente com múltiplas variáveis consideradas possibilitará uma maior área de abrangência na validação da viabilidade do software. Além da seleção dos participantes, serão elaboradas diversas tarefas envolvendo expressões matemáticas de diversos níveis de complexidade.

Todos os testes deverão ser gravados a fim de ser possível considerar não somente questões numéricas como o tempo gasto para a execução de uma tarefa, mas também aspectos comportamentais que indiquem conforto ou inquietação quanto ao software testado.

A aplicação dos testes também contará com a coleta de dados envolvendo as reações emocionais dos usuários utilizando um dispositivo de eletroencefalograma (EEG), o Emotiv EPOC+, de 14 canais. Espera-se verificar o impacto do uso das estratégias de navegação nas tarefas do usuário e em aspectos hedônicos como frustração, estresse e engajamento.

Além da coleta de reações emocionais, após cada tarefa o pesquisador pedirá que os usuários indiquem o nível de dificuldade encontrado na realização da tarefa utilizando uma escala pré-definida, como por exemplo de 1 a 5 onde 1 significa muito difícil e 5 muito fácil.

Análise com implicações para design de materiais interativos com fórmulas matemáticas e implicações para desenvolvimento de sistemas leitores de tela

Os dados dos testes de usabilidade serão analisados por meio de técnicas de análise de conteúdo de vídeo gravados com conteúdo da tela e do próprio usuário no momento da realização das tarefas. A partir disto, serão registrados problemas reportados diretamente pelos usuários durante as sessões e também registrados outros problemas de usabilidade e acessibilidade observados pelos pesquisadores que podem não ter sido reportados diretamente pelos participantes. Serão analisadas também a completude das tarefas, a fim de levantar a taxa de sucesso na execução das tarefas. Os dados sobre dificuldade de 
realização das tarefas e os dados do questionário de satisfação serão usados para a geração de estatísticas sobre satisfação e possíveis considerações futuras passíveis de melhorias.

A análise dos dados coletados pelo dispositivo de eletroencefalograma será feita com software de análise do Emotiv EPOC+ de 14 canais. O software disponibiliza medidas de níveis de engajamento, frustração, empolgação, tédio, stress e meditação. Estes dados serão correlacionados com o desempenho das tarefas nos momentos indicados.

\section{CONTRIBUIÇÕES ESPERADAS}

Espera-se obter através deste estudo o levantamento de dados sobre os métodos atuais de navegação em fórmulas matemáticas, seus pontos fortes e fracos. Através disso, espera-se como principal contribuição ser possível avaliar, elaborar e validar uma nova estratégia de navegação matemática que se apoie nas estratégias convencionais de navegação Web. Com essa nova estratégia espera-se ser capaz de minimizar ou ainda sanar as debilidades encontradas no estado da arte e fortalecer ainda mais os aspectos positivos daquilo que já se tem atualmente.

Como contribuição técnica, espera-se disponibilizar uma versão do leitor de telas ChromeVox com recursos de navegação baseadas em elementos estruturais do MathML através da estratégia elaborada, com leitura em Português do Brasil.

Do ponto de vista científico, espera-se contribuir com o entendimento sobre a relação entre as formas de navegação mais utilizadas por pessoas com deficiência visual em páginas Web e na navegação em conteúdos matemáticos com elementos estruturais. Espera-se ainda retratar como o uso desses recursos pode impactar na redução de esforço e nas reações positivas e negativas na utilização de recursos matemáticos.

\section{AGRADECIMENTOS}

Agradecemos ao CNPq pelo financiamento do projeto NavMatBR e ao Programa de Pós-graduação em Ciência da Computação pelo apoio e financiamento desta pesquisa.

\section{REFERÊNCIAS}

1. Alistair D. N. Edwards, Heather McCartney, and Flavio Fogarolo. 2006. Lambda:: a multimodal approach to making mathematics accessible to blind students. In Proceedings of the 8th international ACM SIGACCESS conference on Computers and accessibility. ACM, 48-54.

2. Helder Ferreira and Diamantino Freitas. 2006. Leitura de Fórmulas Matemáticas para Cegos e Amblíopes: A Aplicação AudioMath. IBERSCAP06 (2006).

3. Pavel Gaura. 2002. REMathEx - Reader and Editor of the Mathematical Expressions for Blind Students. In Computers Helping People with Special Needs, Klaus Miesenberger, Joachim Klaus, and Wolfgang Zagler (Eds.). Springer Berlin Heidelberg, Berlin, Heidelberg, 486-493.

4. Martin Gruber, Jindrich Matousek, Zdenek Hanzlícek, Zdenek Krnoul, and Zbynek Zajíc. 2016. ARET-Automatic Reading of Educational Texts for Visually Impaired Students.. In INTERSPEECH. 383-384.
5. Christopher Power, Helen Petrie, David Swallow, Emma Murphy, Bláithín Gallagher, and Carlos A Velasco. 2013. Navigating, discovering and exploring the web: strategies used by people with print disabilities on interactive websites. In IFIP Conference on Human-Computer Interaction. Springer, 667-684.

6. T.V. Raman. 1994. Audio system for technical readings. Tese de doutorado. Cornell University, Dept. of Computer Science.

7. Luiz F. P. Silva, Antonio A. O. Barbosa, Evelise R. C. G. Freire, Paula C. F. Cardoso, Rafael S. Durelli, and André P. Freire. 2018. Content-based Navigation within Mathematical Formulae on the Web for Blind Users and its Impact on Expected User Effort. In Proceedings of 8th International Conference on Software Development and Technologies for Enhancing Accessibility and Fighting Info-exclusion (DSAI'18).

8. Luiz F P Silva, Otavio F de Oliveira, Evelise R C G Freire, Rosana M Mendes, and André P Freire. 2017. How Much Effort is Necessary for Blind Users to Read Web-based Mathematical Formulae?: A comparison using task models with different screen readers. In Proceedings of the XVI Brazilian Symposium on Human Factors in Computing Systems. ACM, 29.

9. Neil Soiffer. 2005. MathPlayer: Web-based Math Accessibility. In Proceedings of the 7th International ACM SIGACCESS Conference on Computers and Accessibility (Assets '05). ACM, New York, NY, USA, 204-205.

10. Neil Soiffer. 2007. MathPlayer V2.1: Web-based Math Accessibility. In Proceedings of the 9th International ACM SIGACCESS Conference on Computers and Accessibility (Assets '07). ACM, New York, NY, USA, 257-258.

11. Neil Soiffer. 2015. Browser-independent Accessible Math. In Proceedings of the 12th Web for All Conference (W4A '15). ACM, New York, NY, USA, Article 28, 3 pages. DOI : http://dx.doi.org/10.1145/2745555.2746678

12. Volker Sorge, Charles Chen, T. V. Raman, and David Tseng. 2014. Towards Making Mathematics a First Class Citizen in General Screen Readers. In Proceedings of the 11th Web for All Conference (W4A '14). ACM, New York, NY, USA, Article 40, 10 pages.

13. Kátia N. V. Souza. 2010. Alfabetização matemática: considerações sobre a teoria e a prática. Revista de Iniciação Científica da FFC 10, 1 (2010).

14. Robert Stevens, Alistair Edwards, and Philip Harling. 1997. Access to Mathematics for Visually Disabled Students Through Multimodal Interaction. Human-Computer Interaction 12, 1 (1997).

15. Takayuki Watanabe. 2009. Experimental evaluation of usability and accessibility of heading elements. Disability and Rehabilitation: Assistive Technology 4, 4 (2009), 236-247. 